\title{
A COMBINED ATOMIC FORCE MICROSCOPY AND NANOINDENTATION TECHNIQUE TO INVESTIGATE THE ELASTIC PROPERTIES OF BONE STRUCTURAL UNITS
}

\author{
S. Hengsberger ${ }^{1, *}$, A. Kulik ${ }^{2}$ and Ph. Zysset ${ }^{1}$ \\ ${ }^{1}$ Laboratoire de Mécanique Appliquée et d'analyse de Fiabilité and ${ }^{2}$ Institut de Génie Atomique, \\ Ecole Polytechnique Fédérale de Lausanne, Lausanne, Switzerland
}

\begin{abstract}
To our knowledge, this study applied for the first time a recently developed combination of atomic force microscopy (AFM) and nanoindentation on trabecular and compact bone tissue. The major aim was to check the advantage of the available AFM-mode over the conventionally used optical microscope. First, we investigated if removal of the water content helped to prevent enzymatic degradation of the bone tissue and preserve its mechanical properties during a week. After the positive issue of this test, we quantified the intrinsic mechanical properties of single bone structural units (BSU). Bone specimens were obtained from the femoral neck of an 86 year old female. Four BSU were randomly selected and tested each with 24 indents of 5 $\mathrm{mN}$ maximum force.

The available AFM mode proved to be a very useful tool for surface characterization and precise selection of the indentation area. The elastic modulus ranged from $18 \pm 1.7 \mathrm{GPa}$ for a BSU of compact bone to $22.5 \pm 3.1 \mathrm{GPa}$ for a BSU of trabecular bone. Hardness showed values between $0.6 \pm 0.11 \mathrm{GPa}$ for compact bone and $1.1 \pm 0.17 \mathrm{GPa}$ for trabecular bone. The results suggest that the micromechanics of bone tissue may also be described as an assembly of distinct structural units with rather homogeneous material properties.
\end{abstract}

Key Words: Atomic force microscopy, bone structural units, elastic modulus, femur, hardness, nanoindentation.

\footnotetext{
"Address for correspondence

Stefan Hengsberger

LMAF - DGM - EPFL

ME-Ecublens, CH-1015 Lausanne

Switzerland
}

Telephone number: +41216933871

FAX number: +41216933509

E-mail: stefan.hengsberger@epfl.ch

\section{Introduction}

Osteoporosis leads to excessive bone fragility and impairs increasingly the quality of life of the elderly. The deteriorating influences of this metabolic disease on the mechanics of human bone are increasingly well understood in terms of bone density and architecture. Recent studies aimed at quantifying damage accumulation in bone tissue down to the extracellular matrix (ECM) level. It becomes also accepted that the ECM plays the role of a local mechanical sensor providing the cells with the strain or stress information to control the remodeling process and maintain bone tissue. Material characterization of the ECM contributes to the understanding of the biomechanical implications of the normal remodeling process and its perturbation by osteoporosis.

The spectrum of applied experimental techniques to quantify mechanical properties at the bone tissue level range from buckling (Townsend and Rose, 1975), bending (Mente et al., 1989), 3-point-bending (Choi et al., 1990), microtensile and ultrasonic tests (Rho and Ashman, 1993; Ryan and Williams, 1989) to ultrasonic microscopy (Katz and Meunier, 1993). The reported Young's moduli range from $0.76 \mathrm{GPa}$ to $20 \mathrm{GPa}$ for trabecular bone and from 5 GPa to $27 \mathrm{GPa}$ for cortical bone. These values were found to vary strongly with the experimental technique mostly due to distinct strain rates, representative volume element sizes and the associated influence of structural artifacts like lacunae, cement lines and vascular channels. For ultrasonic microscopy, the measured impedance change between the sample and a coupling liquid depends on both Young's modulus and the local material density that cannot easily be distinguished.

More recently, a nanoindentation technique was developed that allows measurement of some mechanical properties within a bone structural unit (BSU). [Note: The bone structural unit, BSU, represents the end result of a remodeling cycle; in cortical bone, it constitutes a Haversian system (or cortical osteon), and in cancellous bone, it is a wall or "packet" of bone (or trabecular osteon) (Eriksen et al., 1994)]. Previous nanoindentation studies (Rho et al., 1997; Zysset et al., 1999; Rho et al., 1999b) characterized the dependence of the elastic modulus of cortical and trabecular bone with age, anatomical site, microstructural orientation and tissue preparation. However, the influence of surface roughness of the tested specimens was not reported. The results by Zysset et al. (1999) for the femoral neck demonstrated standard deviations exceeding $20 \%$ of the mean value, which may be attributed to the inclusion of several bone structural units with quite different properties. The present study focussed on the application of a 
combined atomic force microscopy and nano-indentation technique to measure elastic modulus and hardness of human bone tissue.

The first aim was to check if the preliminary characterization of the surface with the available AFM-mode improves the reliability of the measurements. As a second aim, we investigated if the separate evaluation of single BSUs would give significantly lower standard deviations than $20 \%$ of the mean value.

\section{Technique}

\section{Materials and Methods}

The combination of an AFM and a nanoindenter (Hysitron Incorporated, Minneapolis, MN) is a further development of the traditional Vickers microhardness testing device. This instrument allows for two measurement modes. It provides a surface topography of constant contact force in AFM mode (Figure 1) and a force displacement curve in nanoindentation (Figure 2) mode using the same tip. This feature provides a high spatial resolution to position the tip on the microstructure of interest. The sample is mounted on a scanner that allows for a movement in the plane normal to the axial motion of the tip. The transducer consists of a three-plate capacitor on whose central plate a tetrahedral diamond Berkovich-tip is mounted. A nanoindentation curve (see Figure 2) consists of a loading phase where the tip is pressed into the material up to a maximal force, a holding period where the tip creeps into the material and an unloading phase where the force on the material is released. The loading and holding phases result in both plastic and elastic deformation that cannot be distinguished. The unloading phase shows the elastic recovery of the material while the load is released. For isotropic elastic materials, the following relationship based on the analytical solution by Sneddon (1965) holds for indenters of revolution:

$$
S\left(h_{\max }\right)=\frac{d P}{d h}\left(h_{\max }\right)=\frac{2}{\sqrt{\pi}} E_{r} \sqrt{A_{c}\left(h_{\max }\right)}
$$

where $\mathrm{S}\left(\mathrm{h}_{\max }\right)$ describes the derivative of the unloading part at the point of initial unloading and $\mathrm{A}_{\mathrm{c}} \mathrm{h}_{\max }$ the contact area, over which the material and the indenter are in contact at that position and time. The unloading stiffness $\mathrm{S}\left(\mathrm{h}_{\max }\right)$ is determined by fitting the unloading curve between $40 \%$ and $95 \%$ of maximum force. The contact area $\mathrm{A}_{\mathrm{c}} \mathrm{h}_{\max }$ is determined by a procedure derived by Oliver et al. (1992). The calibration of the instrument is commonly performed by doing indents of increasing depth in fused silica with a known reduced modulus of $69.9 \mathrm{GPa}$. The reduced modulus $\mathrm{E}_{\mathrm{r}}$ depends on the deformation of the material and the deformation of the diamond tip:

$$
\frac{1}{E_{r}}=\frac{1-v_{\text {specimen }}^{2}}{E_{\text {specimen }}}+\frac{1-v_{\text {tip }}^{2}}{E_{\text {tip }}}
$$

The material properties of the diamond tip are $v_{\text {tip }}=0.07$ and $\mathrm{E}_{\text {tip }}=1140 \mathrm{GPa}$. The indentation modulus is defined by

$$
E_{\text {ind }}=\frac{E_{\text {specimen }}}{1-v_{\text {specimen }}^{2}}
$$

This variable contains Young's modulus and Poisson's ratio. Often, Poisson's ratio is assumed to be $v=0.3$ for

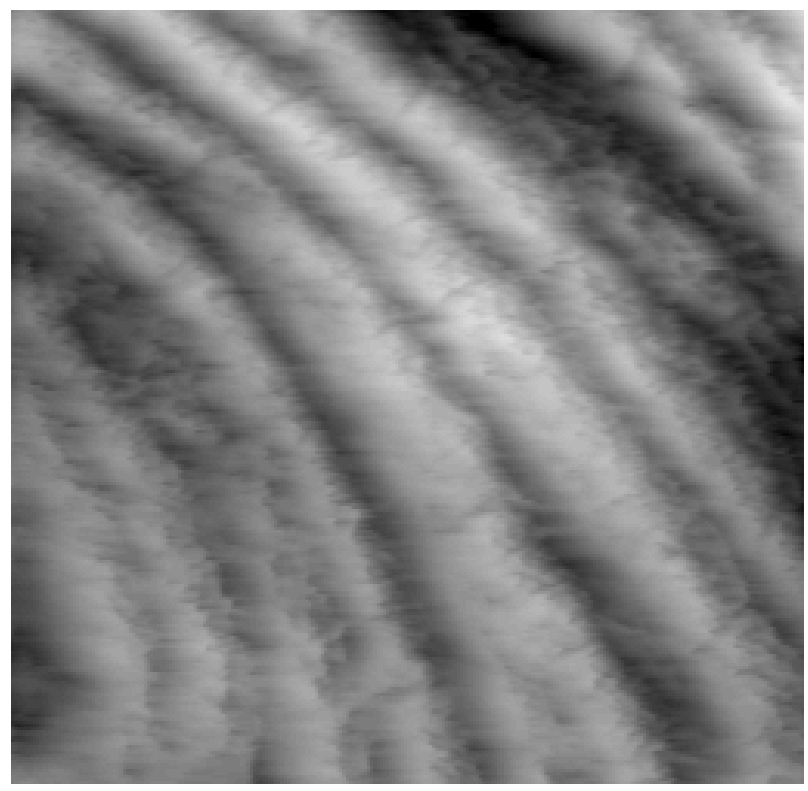

Figure 1. Topography of compact bone in AFM-mode (scan size $50 \mu \mathrm{m}$ x $50 \mu \mathrm{m}$ ).

bone tissue, but we prefer to report indentation moduli to avoid this assumption. For the purpose of comparison, Young's modulus calculated for $v=0.3$ is approximately $90 \%$ of the indentation modulus.

A second mechanical property can be calculated from the nanoindentation curve: hardness. This variable describes the mean pressure the material can resist and is defined by the ratio of maximum load $\mathrm{P}_{\text {max }}$ over the contact area:

$$
H=\frac{P_{\max }}{A_{c}\left(h_{\max }\right)}
$$

The stress field imposed by the indentation process is heterogeneous and leads to plastic deformation around the tip. To achieve reproducible measurements, the average roughness of the sample in the contact area should be well below the applied indentation depth. The minimal distance between neighbor indents was set to approximately 3 times the contact diameter (i.e. $\approx 18 \mathrm{~h}_{\text {max }}$ for a Berkovich tip geometry).

\section{Specimen preparation and measurements:}

The femoral head of an 86 year old female was cut in $3 \mathrm{~mm}$ slices perpendicular to the axis of the neck. For this purpose, a diamond band saw with continuous water irrigation was employed. The marrow was dissolved applying about 3 alternated treatments with $0.5 \%$ bleach and ordinary soap solution in an ultrasonic cleaner for $5 \mathrm{~min}$ utes (Zysset et al. 1994). The specimen was then embedded in PMMA without fixation to prevent possible deterioration of the mechanical properties (McElhaney et al., 1964, Sedlin and Hirsch, 1966). The sample was then polished, first with successive grades of silicon carbide paper, followed by a treatment with diamond slurry and finishing with a $0.05 \mu \mathrm{m}$ alumina solution. The water content of bone promotes the enzymatic degradation of collagen and reduction in mechanical properties within several hours at ambient temperature. Since characterization of each BSU took at least one entire day, it was necessary to stabilize the mechanical properties for several days with an additional drying procedure for 24 hours at $50^{\circ} \mathrm{C}$. After 


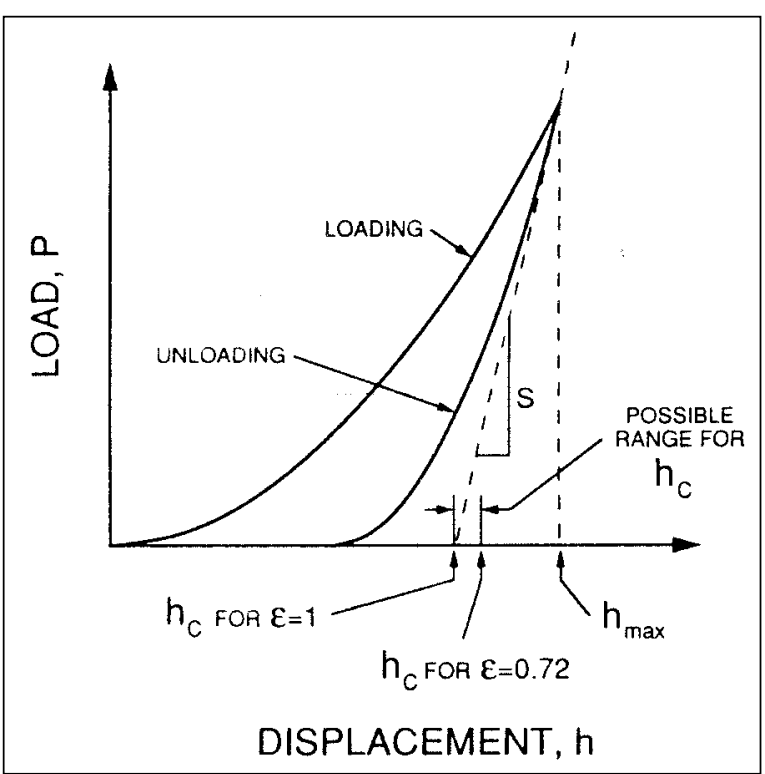

Figure 2. Typical nanoindentation curve. The unloading part allows to determine elastic modulus and hardness.

drying the sample was first scanned in AFM-mode with a typical size of $60 \mu \mathrm{m}$ times $60 \mu \mathrm{m}$, a frequency of $0.3 \mathrm{~Hz}$ and a contact force of $5 \mu \mathrm{N}$. The topography measurement helped to characterize the polishing quality and to choose precisely the region of interest. The piezoelectric scanner of the AFM allows for a higher positioning accuracy than the $\mathrm{x}-\mathrm{y}$ translation table that equips nanoindentation devices relying on optical microscopy. First, we tested if the high contact force of the tip damaged the specimen surface. For this purpose several successive scans with the same center and increasing scan size were performed. Then, 3 indents of $2.5 \mathrm{mN}$ maximum force on the same osteon were done each day during a period of one week. The purpose was to check if the mechanical properties remained constant over time. After these preliminary tests, two BSU in cortical and two BSU in trabecular bone were tested. The tests included 24 indents in each BSU with a maximum force of $5 \mathrm{mN}$. The load-controlled indentations reached depths of about $550 \mathrm{~nm}$ that varied in correlation to the stiffness of the tested region. The available scanning option enabled us to correct slight tilts between the samples' surface normal and the transducer axis for each individual indent. All tests included a preliminary thermal drift correction limited to a maximum drift of $0.05 \mathrm{nms}^{-1}$. The indentations contained a linear loading part of $15 \mathrm{~s}$, a $10 \mathrm{~s}$ holding period at maximum force and a linear unloading part of $15 \mathrm{~s}$. These parameters correspond to an average strain rate of

$$
\frac{1 d h}{h d t} \approx 0.067 \frac{1}{s}
$$

According to the orientation of the femur section and for best identification of the BSU, the indents were applied parallel to the osteon axes in compact bone. On cancellous bone the indents were applied perpendicular to the long
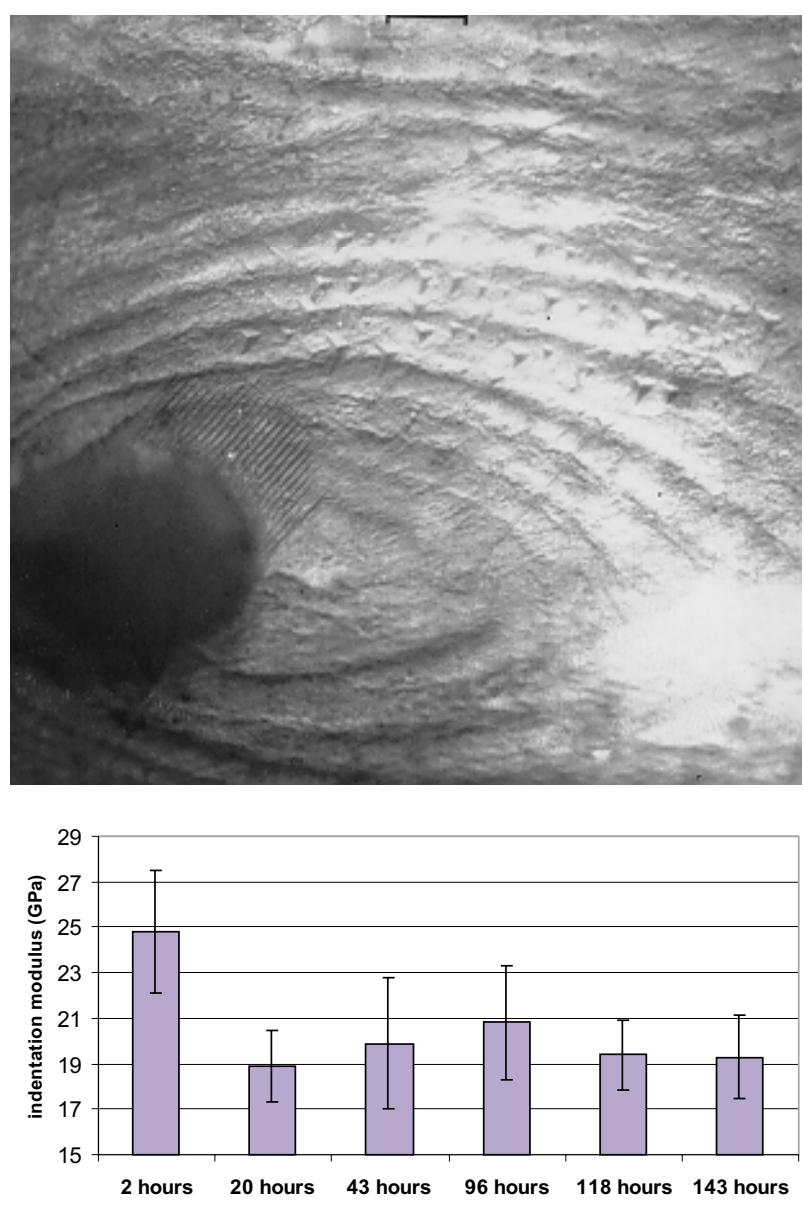

Figure 3. (a) Optical image of an osteon after data acquisiion. The triangular marks are the remaining imprints of the indents. (b) Time dependence of the indentation modulus of compact bone after drying. After one day, the indentation modulus reached a stabilized level and did not change in a significant way any more.

axes of transversely orientated trabeculae.

\section{Results}

\section{AFM}

The successive topography measurements with increasing scan size showed that the selected contact force of $5 \mu \mathrm{N}$ did not affect the surface appearance and topography. After polishing, bone lamellae showed an excellent contrast in the AFM-scan. Those lamellae that appeared bright under AFM (peaks) corresponded to the optically bright lamellae and similarly, the dark lamellae (valleys) corresponded to the optically dark lamellae. Neighboring lamellae showed typical height differences on the order of $60 \mathrm{~nm}$ but could range from 20 to $140 \mathrm{~nm}$. The average roughness, calculated for the entire scan area, was typically between $30 \mathrm{~nm}$ and $100 \mathrm{~nm}$.

\section{Effect of drying}

The indentation modulus was highest immediately after drying and was decreased by about $20 \%$ within the next 24 hours. The following days, the modulus (see Figure 3) did not change in a significant way $(p>0.05)$. The standard deviations of the elastic moduli (error bars) within single BSU were approximately $10 \%$. A similar evolution of the mechanical properties after drying could be repro- 


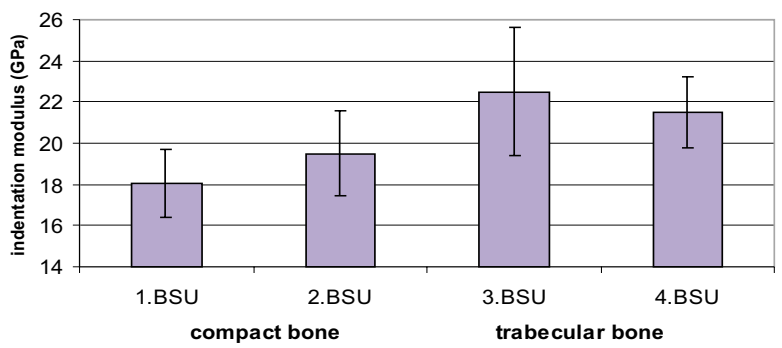

Figure 4. Average indentation modulus of $5 \mathrm{mN}$ indents for the 4 tested BSU. The variations are most probably due to differences in mineral content.

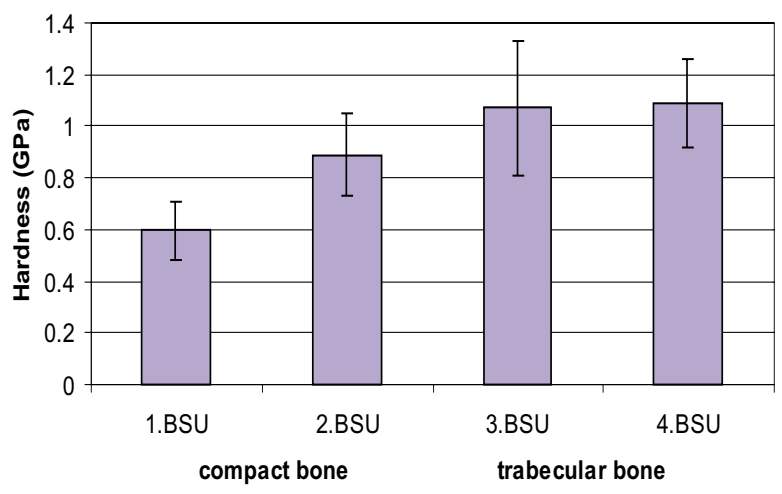

Figure 5. Hardness values of $5 \mathrm{mN}$ indents for the 4 BSU. Variations can be seen with trends comparable to the indentation modulus.

duced for trabecular bone. The hardness followed the same trend and changed from $1.2 \pm 0.15 \mathrm{GPa}$ to $0.9 \pm 0.2 \mathrm{GPa}$ within the first 24 hours and became stable. The right picture in Figure 3 shows an osteon after data acquisition. The location of the indentations can be recognized as triangular imprints. The effect of decreasing indentation modulus after the first day may be attributed to rehydration of the bone tissue under the normal humidity conditions of the laboratory.

\section{Test of four BSUs}

The graph in Figure 4 represents the mean indentation moduli of $550 \mathrm{~nm}(5 \mathrm{mN})$ indents for the 4 BSUs. The error bars indicate standard deviations. The indentation modulus ranged from $18 \pm 1.7 \mathrm{GPa}$ in compact bone to 22.5 $\pm 3.1 \mathrm{GPa}$ in trabecular bone. According to our ANOVA analysis, the mean indentation modulus of the 4 BSU was significantly different $(p<0.0001)$. The two BSU of compact bone showed also significantly different indentation moduli $(\mathrm{p}<0.01)$. The latter effect is most probably due to differences in mineralization (Currey, 1969). The nanoindentation parameters of the $5 \mathrm{mN}$ indents correspond to those applied by Zysset et al. (1999) who investigated eight human femoral heads under moist conditions. According to this study the indentation moduli were $12.5 \pm 6.15$ $\mathrm{GPa}$ for trabecular bone and 17.4 $\pm 5.8 \mathrm{GPa}$ for compact bone. Surprisingly, the elastic properties of trabecular bone were found to be higher than those of compact bone in the present study. Due to the very limited statistics, we attribute this finding to the random selection of the four BSU. The standard deviations of the indentation moduli are within
$10 \%$ of the mean value and therefore substantially lower than previous studies including several BSU for each donor (Zysset et al., 1999).

The hardness values for the four tested BSUs showed similar trends as the indentation moduli (Fig. 5). This observation compares favorably with the microindentation study by Evans et al. (1990) and the nanoindentations performed by Rho and Pharr (1999), where a relatively high correlation between elasticity and hardness was found $\left(\mathrm{r}^{2}=0.96\right.$ given by Evans et al. $)$. The hardness ranged from $0.6 \pm 0.11 \mathrm{GPa}$ for compact bone to $1.1 \pm 0.17 \mathrm{GPa}$ for trabecular bone. For moist conditions, Zysset et al. (1999) found averages between $0.234 \mathrm{GPa}$ for trabecular bone and $0.76 \mathrm{GPa}$ for compact bone.

\section{Discussion}

The combination of AFM and nanoindentation has proven to be a powerful tool to provide reliable micromechanical properties of bone tissue. The strong advantage of the available AFM-mode over the conventional nanoindentation technique lies in the possibility to select the location of the indentations within a few tenths of nanometers and to quantify the sample's surface topography, hence the quality of the sample preparation.

Drying the bone specimens for 24 hours at $50^{\circ} \mathrm{C}$ increased but conserved the mechanical properties for several days. BSUs from the same anatomical site of the same donor and undergoing the same preparation process showed significant variations in mean mechanical properties that may be attributed to differences in mineral content. The higher standard deviations found in previous nanoindentation studies were probably associated with averaging over multiple BSU and/or donors. The influence of nanoindentation parameters on bone results should therefore be determined within the same BSU.

This study allowed comparing several BSU from the femoral neck of a single donor. The results indicated that, in first approximation, bone might be seen as an assembly of structural units with distinct mechanical properties between them but rather homogeneous properties within the same BSU. This finding has potentially important implications in the process of fracture propagation that is clearly related to tissue heterogeneity.

The major limitation of the nanoindentation data analysis remains the hypothesis of isotropy of the tested material. In fact, the indentation curve depends to a widely unknown extent on all anisotropic elastic constants of the tested material. Since bone tissue and most probably also the bone ECM (Rho et al., 1999a) is elastically anisotropic, the reported results are some weighted average of the elastic moduli along the various material orientations.

\section{References}

Choi K, Kuhn JL, Ciarelli MJ, Goldstein SA (1990) Elastic moduli of human subchondral trabecular and cortical bone tissue and the size-dependency of cortical bone modulus. J Biomech 23: 1103-1113.

Currey JD (1969) The mechanical consequences of variation in the mineral content of bone. J Biomech 2: 1- 
11.

Eriksen EF, Axelrod DW, Melsen FM (1994) Bone Histomorphometry, 1st ed. Raven Press, New York.

Evans GP, Behiri JC, Currey JD, Bonfield W (1990)

Microhardness and Young's modulus in cortical bone exhibiting a wide range of mineral volume fractions, and in a bone analogue. J Mat Sc: Mat Med 1: 38-43.

Katz JL, Meunier A (1993) Scanning acoustic microscope studies of the elastic properties of osteons. J Biomech Eng 115: 543-548.

McElhaney J, Fogle J, Byars E Weaver G (1964) Effect of embalming on the mechanical properties of beef bone. J Appl Physiol 19: 1234-1236.

Mente PL, Lewis JL (1989) Experimental method for the measurement of the elastic modulus of trabecular bone tissue. J Orthopaed Res 7: 456-461.

Oliver WC, Pharr GM (1992) An improved technique for determining hardness and elastic modulus using load and displacement sensing indentation experiments. Mat Res Soc 7: 1564-1583.

Rho JY, Ashman RB (1993) Young's modulus of trabecular and cortical bone material: ultrasonic and microtensile measurements. J Biomech 26: 111-119.

Rho JY, Pharr GM (1999) Effects of drying on the mechanical properties of bovine femur measured by nanoindentation. J Mat Sc: Mat Med 10: 485-488.

Rho JY, Tsui YT, Pharr GM (1997) Elastic properties of human cortical and trabecular lamellar bone measured by nanoindentation. Biomaterials 18: 1325-1330.

Rho JY, Roy ME, Tsui TY, Pharr GM (1999a) Elastic properties of microstructural components of human bone tissue as measured by nanoindentation. J Biomed Mat Res 45: 48-54.

Rho JY, Roy ME, Tsui TY, Evans, ND, Pharr GM (1999b) Mechanical and morphological variation of the human lumbar vertebral cortical and trabecular bone. J Biomed Mat Res 44: 191-199.

Ryan SD, Williams JL (1989) Tensile testing of rodlike trabeculae excised from bovine femoral bone. $\mathrm{J}$ Biomech 26: 77-83.

Sedlin ED, Hirsch C (1966) Factors affecting determination of the physical properties of femoral cortical bone. Acta Orthop Scand 37: 29-48.

Sneddon IN (1965) The relation between load and penetration in the axisymmetric Boussinesq problem for a punch of arbitrary profile. Int J Eng Sc 3: 47-57.

Townsend PR, Rose RM (1975) Buckling study of single human trabeculae. J Biomech 8: 199-201.

Zysset P, Sonny M, Hayes WC (1994) Morphologymechanical property relations in trabecular bone of the osteoarthritic proximal tibia. J Arthroplasty 9: 203-216.

Zysset PK, Guo XE, Hoffler CE, Moore KE, Goldstein SA (1999) Elastic modulus and hardness of cortical and trabecular bone lamellae measured by nanoindentation in the human femur. J Biomech 32: 1005-1012.

\section{Discussion with Reviewers}

S. Weiner: Did the authors find any systematic trend with increasing distance from the lamellar boundary plane?

Authors: We did not examine the dependence of indentation modulus with increasing distance to the lamellar boundary because single lamellae are not sufficiently large to position several neighboring indents. We did observe a dependence of the indentation modulus with depth that is described in a paper that is currently submitted elsewhere.

S. Weiner: Error obtained for repeated measurements should decrease with increasing indent size.

Authors: Surprisingly, our standard deviations were not influenced by indentation depth, which we attribute to the high force and displacement sensitivity of our instrument and the minimal roughness of the tested surface selected on the AFM scan.

S. Weiner: Anisotropy is not a probable property of lamellar bone but a certainty.

J. Currey: There is no doubt that bone is anisotropic, many, many studies have shown this.

Authors: We agree. From a mechanical standpoint, we distinguish the anisotropy of the ECM from the anisotropy of bone at the macroscopic level ( $\mathrm{mm})$ that includes oriented vascular channels and lacunae. However, the studies by Ziv et al. (1996) and Rho et al. (1999b) provide experimental evidence for mechanical anisotropy also at the ECM level.

J. Currey: Indents parallel to the osteon axes will be parallel to the grain of the bone. On the other hand indents perpendicular to the transversely orientated trabeculae will be normal to the grain of the bone. Why have authors chosen a different orientation for the two tissue types?

J.Y. Rho: Indentations were made in only one plane in the cortical and the trabecular bone surfaces. What was the rationale behind choosing those planes over the other planes? Given the anisotropic nature of bone, would data in planes transverse to those tested be worth obtaining. Finally, it is clear which plane the cortical bone specimen was indented, relative to the long axis. Please be more explicit with regard to which plane was indented in the trabecular bone, relative to the long axis.

Authors: The grain of a trabecular BSU can be defined by the normal of the lamellae and the axis of the trabeculae, but to our knowledge there is no evidence that the ECM ultrastructure of the trabecular BSU will be identical to that of a compact BSU in their respective grain coordinate system. In fact, the ECM ultrastructure seems even to vary within compact bone and motivate a classification of osteons as longitudinal, alternate and transverse.

In practice, the directions defining trabecular grain are difficult to determine. The identification of the contours of a single BSU in the transverse section of a trabecula is also difficult. Since the goal of this study was not to compare compact and trabecular bone tissue, we tested the BSU along the most accessible direction that belonged to the 
plane of the lamellae for both tissues.

J.Y. Rho: A bleach solution will affect the collagen in bone. In general, these solutions have been used for decollagenization. Will this cause a serious deterioration of the mechanical properties of bone?

Authors: The bleach concentration of $0.5 \%$ used in our study is extremely low and this protocol was not found to degrade the physical properties of human cancellous bone in Zysset et al. (1994). Since the sensitivity of mechanical data to biochemical treatments is probably increased when testing a superficial layer of tissue, we selected the indentation locations at least $30 \mu \mathrm{m}$ away from the bone edge.

J.Y. Rho: “...rather homogenous properties within the same BSU": This suggestion is not supported from the re- sults of the present study, because as the authors mentioned the elastic properties are significantly different between a BSU of compact bone $(18.1 \pm 1.7 \mathrm{GPa})$ and a BSU of trabecular bone $(22.5 \pm 3.1 \mathrm{GPa})$.

Authors: BSU was a significant global factor for all tested units, but indeed the two trabecular bone units were not significantly different according to the pairwise comparison of means. The other 5 pairs were significantly different, which supports our statement.

\section{Additional Reference}

Ziv V, Wagner HD, Weiner S (1996) Micro-structuremicrohardness relations in parallel-fibered and lamellar bone. Bone 18: 417-428. 